\title{
Diagnosis, complications and management of chronic neutrophilic leukaemia: A case report
}

\author{
PATRÍCIA ROCHA SILVA ${ }^{1}$, CRISTINA FERREIRA ${ }^{1}$, SUSANA BIZARRO $^{2}$, NUNO CERVEIRA $^{2}$, \\ LURDES TORRES ${ }^{2}$, ILÍDIA MOREIRA ${ }^{1}$ and JOSÉ MÁRIO MARIZ ${ }^{1}$
}

Departments of ${ }^{1}$ Oncohaematology and ${ }^{2}$ Genetics, The Francisco Gentil Portuguese Institute of Oncology, Porto 4200-072, Portugal

Received July 2, 2014; Accepted March 9, 2015

DOI: $10.3892 / \mathrm{ol} .2015 .3148$

\begin{abstract}
Chronic neutrophilic leukemia (CNL) is a rare myeloproliferative neoplasm characterized by sustained neutrophilia and the absence of the Philadelphia chromosome or the $B C R-A B L 1$ fusion gene. The present study reports the case of a 59-year-old Caucasian female that was referred to The Francisco Gentil Portuguese Institute of Oncology (Porto, Portugal) with constitutional symptoms (mainly asthenia), marked leukocytosis $\left(51.33 \times 10^{9} / 1\right.$ with $90 \%$ neutrophils), macrocytic anemia and splenomegaly. Bone marrow aspiration and biopsy revealed hypercellular marrow with clear predominance of segmented neutrophils. The karyotype was normal and the $B C R-A B L 1$ fusion gene was not detected. After excluding a leukemoid reaction, a diagnosis of CNL was established. The clinical follow-up was complicated by hemorrhagic brain lesions and relapsing episodes of erythematous, well-demarcated and painful subcutaneous nodular lesions, consistent with Sweet's syndrome (SS). Multiple treatment strategies were administered, including use of hydroxyurea, imatinib and intensive chemotherapy. Nevertheless, progression was documented and the patient succumbed at 28 months post-diagnosis. The clinical course of CNL varies, and can be complicated by cerebral hemorrhage, blastic transformation or infection. Dermatological manifestations such as SS have seldom been reported in association. No evidence-based treatment currently exists and the majority of our knowledge is based on results from case reports and small series.
\end{abstract}

Correspondence to: Dr Patrícia Rocha Silva, Department of Oncohaematology, The Francisco Gentil Portuguese Institute of Oncology, Rua Doutor António Bernardino de Almeida, Porto 4200-072, Portugal

E-mail: patricia.rocha.silva81@gmail.com

Key words: chronic neutrophilic leukaemia, myeloproliferative disorders, atypical chronic myeloid leukaemia, Sweet's syndrome

\section{Introduction}

Chronic neutrophilic leukemia (CNL) is a rare myeloproliferative neoplasm characterized by sustained mature neutrophilic leukocytosis with few or no circulating immature granulocytes, hepatosplenomegaly and hypercellular bone marrow with a predominance of myeloid mature cells. In addition, no Philadelphia chromosome or $B C R-A B L 1$ fusion gene are detected. Generally affects older adults and both genders $(1,2)$. The cause of CNL is not known (1). The majority of patients have a poor prognosis, with a median survival of $<3$ years (3). Treatment has consisted largely of cytoreductive agents such as hydroxyurea, with an allogenic bone marrow transplant being the only curative treatment (4).

The present study reports the case of a patient with CNL complicated with Sweet's syndrome (SS) and hemorrhagic brain lesions. The study also describes the multiple therapeutic strategies and the difficulties in managing this disease.

\section{Case report}

Presentation and diagnosis. A 59-year-old Caucasian female, with a past history of hypertension, was referred to The Francisco Gentil Portuguese Institute of Oncology (Porto, Portugal) in October 2010, presenting with asthenia, shortness of breath, abdominal pain, pallor, splenomegaly $(6 \mathrm{~cm}$ below the costal margin) and profuse lower limb oedema. Laboratory analysis showed macrocytic anemia and leukocytosis [haemoglobin level, $8.4 \mathrm{~g} / \mathrm{dl}$ (normal range, 11.5-16.5 g/dl); mean cell volume, $117.3 \mathrm{fl}$ (normal range, 76.0-96.0 fl); white blood cell count, $51.33 \times 10^{9} / 1$ (normal range, 4.0-11.0x10 $/ 1$ ); with $90 \%$ neutrophils; and platelet count, $173 \times 10^{9} / 1$ (normal range, 150-400×10\%/1); a raised lactate dehydrogenase level, 392 U/1 (normal range, 67-248 U/l); and a raised vitamin B12 level, >2000 pg/ml (normal range, 191-663 pg/ml)] and mild hepatic dysfunction. Bone marrow aspiration revealed hypercellular marrow with a clear predominance of segmented neutrophils, few immature granulocytes and no myeloblasts (Fig. 1). The biopsy confirmed these findings plus the presence of diffuse reticulin fibrosis. The karyotype was normal and the BCR-ABLI and BCR-PDGFRA fusion genes were not detected. Mutation analysis of the JAK2,MPL and CALR genes was negative. Causes of leukemoid reaction, including other 
malignancies, severe infections or inflammatory conditions, drugs, severe hemorrhage or acute hemolysis were excluded based on obtaining a detailed clinical history, physical examination, imaging and laboratory studies, and the diagnosis of CNL was determined.

Treatment and evolution. Cytoreduction with hydroxyurea $(1,500 \mathrm{mg} /$ day $)$ was started, resulting in initial hematological improvement. One month later, fever and erythematous, well-demarcated and painful subcutaneous nodular lesions developed in the right breast, right upper limb and suprapubic region. These symptoms were reversed following administration of ceftriaxone ( $1 \mathrm{~g} /$ day; duration, 3 days) followed by 14 days of levofloxacin $(500 \mathrm{mg} /$ day $)$ and suspension of hydroxyurea. Meanwhile, disease progression was documented with increasing splenomegaly (10 $\mathrm{cm}$ below the costal margin), peripheral blood neutrophilia $\left(12.08 \times 10^{9} / 1\right.$; normal range, 2.0-7.5 x10 $/ \mathrm{l}$ ), anaemia (haemoglobin $7.7 \mathrm{~g} / \mathrm{dl}$; normal range, $11.5-16.6 \mathrm{~g} / \mathrm{dl}$ ) and the development of deep vein thrombosis of the left lower limb. Intensive treatment with acute leukemia induction-like chemotherapy was proposed, but was postponed due to fever and profuse new skin lesions. The largest lesion $(15 \times 10 \mathrm{~cm})$, located in the right thigh, was ulcerated with extensive loss of skin tissue. A skin biopsy revealed predominantly lobular neutrophilic panniculitis compatible with SS. Improvement was achieved with prednisolone $(1 \mathrm{mg} / \mathrm{kg})$ and amoxicillin and clavulanic acid (875/125 $\mathrm{mg} / \mathrm{t}$ wice per day) for a duration of 2 weeks. Upon steroid administration, a reduction in spleen size ( $3 \mathrm{~cm}$ below the costal margin) and recovery of the hematological values were observed, and a partial hematological response was achieved with low doses of corticosteroids (40 mg/day) and hydroxyurea (500 mg/day). Six months later, while the skin lesions were recovering, worsening of the anemia, thrombocytopenia and splenomegaly $(20 \mathrm{~cm}$ below costal margin) led to the administration of $400 \mathrm{mg} / \mathrm{day}$ imatinib for one month. No hematological improvement was observed and the patient required blood transfusions. When intensive chemotherapy was finally proposed at 15 months post-diagnosis, the patient presented with a mild headache and gait imbalance. Brain computed tomography and magnetic resonance imaging were performed, showing multiple hemorrhagic lesions, and the hypothesis of cerebral nervous system invasion by CNL was consequently considered. The lumbar puncture, even without trauma, showed a persistently hematic and neutrophilic cerebrospinal fluid, with negative cultures. Intensive chemotherapy with high-dose cytarabine, daunorubicin and cyclosporine (protocol SWOG 9126) (5) was administered for two cycles. During treatment, several infectious complications and another set of erythematous skin lesions were observed (Fig. 2). The skin lesions were resolved using corticosteroids ( $1 \mathrm{mg} / \mathrm{kg} /$ day; duration, 2 weeks); and large spectrum antibiotic treatment (meropenem, amikacin, vancomycin and voriconazol) was concomitant. The final histology was inconclusive. Following the chemotherapy, the spleen was reduced in size, the cytopenia was improved and the neurological symptoms were resolved, along with the brain imaging changes.

No siblings were available for allogeneic hematopoietic progenitor transplant, so this treatment could not be considered, and the disease was therefore only controlled for four

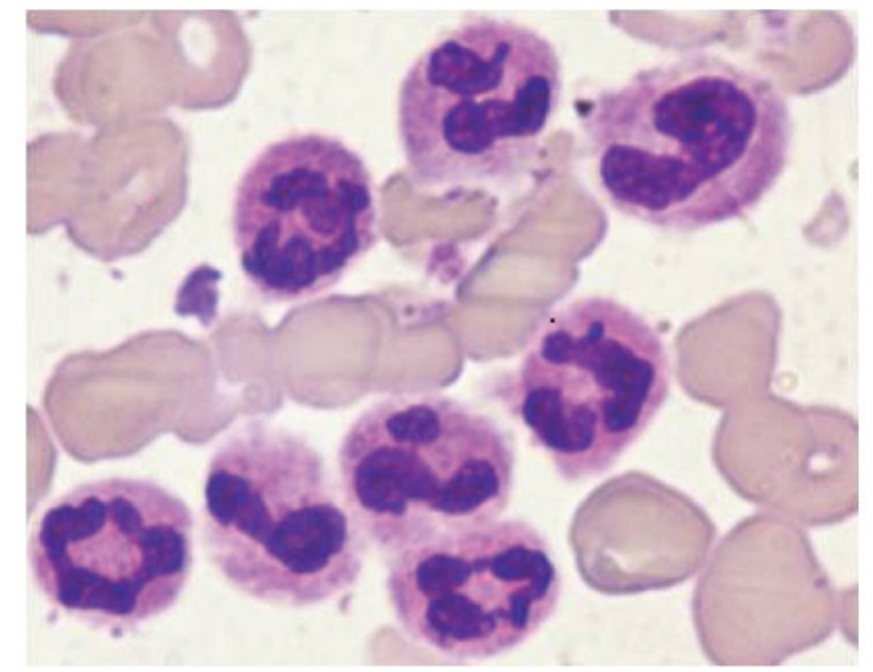

Figure 1. Bone marrow aspirate smear demonstrating neutrophilic proliferation (Wright-Giemsa staining; original magnification, x1,000).

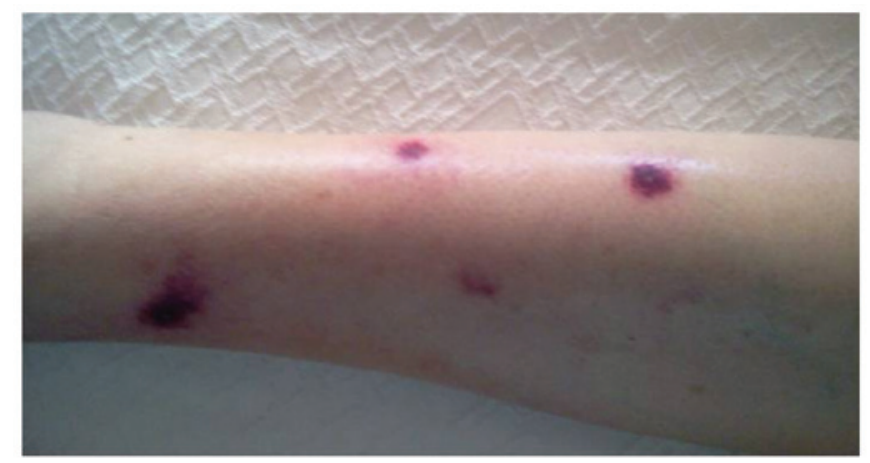

Figure 2. Erythematous, well-demarcated nodular lesions in the right leg consistent with Sweet's syndrome.

months. When progression was documented, best supportive and palliative care was instituted with oral cytoreduction and transfusion support. The patient succumbed at 28 months post-diagnosis.

\section{Discussion}

The first case of CNL was described in 1920 by Tuohy (6), and since then, not much has been elucidated with regard to its pathogenesis and treatment due to the rarity of this myeloproliferative neoplasm. CNL equally affects the two genders and is more common in the elderly population ( $>60$ years) $(1,4)$. In total, $\sim 150$ cases have been described (1), however, a review performed in 2006 indicated that only 40 cases met the rigid criteria of the World Health Organisation classification (7). In the present study, CNL was considered following the proper exclusion of secondary causes of neutrophilia, such as occult malignancy, ongoing infections and other inflammatory conditions (8). The diagnosis was based on peripheral blood leukocytosis with neutrophilia, the presence of hepatosplenomegaly, hypercellular bone marrow with a predominance of myeloid lineage (mature forms), and the absence of the $B C R-A B L 1$ fusion gene and the Philadelphia chromosome. It was essential to exclude 
$B C R$ - $A B L 1$-positive chronic myelogenous leukemia (CML), the main variant that encodes the p230 protein, since similar to CNL, the condition exhibits peripheral blood neutrophilia. Although $B C R$ - $A B L 1$-positive CML was previously considered to be a form of CNL $(9,10)$, it is now considered a form of CML (2). The BCR-PDGFRA rearrangement and the JAK2 and $C A L R$ mutations were also tested for in the present study. These mutations have been identified in myeloproliferative disorders, but only $\mathrm{J} A K 2$ has been reported in CNL (11-13). Recently, Maxson et al reported the association between receptor for colony-stimulating factor 3 (CSF3R) mutations and CNL and atypical CML (14), which could be a promising marker of the disease (15). In the present case, the karyotype was normal, but nearly $10 \%$ of CNL cases are associated with cytogenetic abnormalities, including trisomy 8, 9, 21 and deletion of 11(q), $12(\mathrm{p}), 17(\mathrm{p})$ and 20(q), the latter possibly being associated with a more favorable prognosis (16-18).

SS, a neutrophilic dermatosis characterized by the development of erythematous, tender plaques or nodules frequently with fever and leukocytosis, has been associated with hematological malignancies $(19,20)$. The differential diagnosis is wide and includes infectious disorders, as well as neoplastic conditions such as leukemia cutis (19). To the best of our knowledge, only two cases of SS have previously been reported in association with CNL $(21,22)$. In the present patient, SS was histological confirmed following a second febrile episode of cutaneous lesions, and was successfully treated with prednisolone. Corticosteroids are the standard treatment for this condition, but antibiotics may assist, particularly when lesions became secondarily infected. Spontaneous remissions can occur (19); this may explain the apparent response to antibiotics in the first episode (although this was not histological-proven $\mathrm{SS}$ ) and justified their use on the other relapses.

During disease progression, the patient presented with neurological symptoms caused by brain hemorrhagic lesions. In CNL, two possible causes may be responsible for this: Invasion of the central nervous system and/or thrombocytopenia. While thrombocytopenia and abnormal platelet function have been reported as causes of the bleeding tendency in CNL $(23,24)$ by certain studies, others have demonstrated either invasion and mass formation of neutrophils in the brain parenchyma upon autopsy (25), or extensive infiltration of mature and immature neutrophils, resulting in the destruction of vascular walls (26). Based on the absence of other systemic hemorrhagic manifestations in the present study, these last explanation appear more likely.

There is no standard therapy for CNL. Hydroxyurea has been shown to control the disease temporarily, while intensive chemotherapy confers an increased risk of mortality by infection or hemorrhage (27). The only curative treatment is allogeneic bone marrow transplant, however, the age of the majority of CNL patients excludes this therapeutic approach (28). In the present case, hydroxyurea was efficient for less than one year, and imatinib treatment was attempted one month with no response. This selective tyrosine kinase inhibitor has proven valid against atypical myeloproliferative neoplasms harboring mutations in the $A B L 1, K I T$ and platelet-derived growth factor receptor (PDGFRB or PDGFRA) genes $(29,30)$. In the absence of an identified molecular marker, the success of this therapeutic approach in anecdotal reports is believed to be due to unknown molecular mechanisms. The study by Choi et al reported the case of a CNL patient carrying $\mathrm{t}(15 ; 19)(\mathrm{q} 13 ; \mathrm{p} 13.3)$ in which cytogenetic remission was achieved following daily treatment with $400 \mathrm{mg}$ imatinib (31). In the present case, the two cycles of intensive chemotherapy resulted in a short partial response of four months.

The overall prognosis of patients with CNL is poor, with a median survival time of 30 months $(3,7)$. Death is usually due to cerebral hemorrhage, blastic transformation or fulminant infection $(32,33)$. In spite of therapeutic intervention, the present patient followed the natural history of the disease. The rarity of CNL makes clinical trials unlikely to be conducted, and the majority of our knowledge is based on case reports and small series. Novel genetic markers, such as CSF3R, will provide a better understanding of this disorder, and provide novel diagnostic tools and therapeutic targets.

\section{References}

1. Bain B, Brunning R and Vardiman J: Chronic neutrophilic leukaemia.In: WHO Classification of Tumours of Haematopoietic and Lymphoid Tissues. Swerdlow S, Campo E and Harris NL (eds). IARC Press, France, pp38-39, 2008.

2. Vardiman J and Hyjek E: World health organization classification, evaluation and genetics of the myeloproliferative neoplasm variants. Hematology Am Soc Hematol Educ Program 2011: 250-256, 2011.

3. Reilly JT: Chronic neutrophilic leukaemia: a distinct clinical entity? Br J Haematol 116: 10-18, 2002.

4. Bohm J and Schaefer HE: Chronic neutrophilic leukaemia: 14 new cases of an uncommon myeloproliferative disease. J Clin Pathol 55: 862-864, 2002.

5. List AF, Kopecky KJ, Qillman CL, et al: Benefit of cyclosporine modulation of drug resistance in patients with poor-risk acute myeloid leukemia: a Southwest Oncology Group study. Blood 98: 3212-3220, 2001

6. Tuohy EL: A case of splenomegaly with polymorphonuclear neutrophil hyperleukocytosis. Am J Med Sci 160: 18-25, 1920.

7. Elliott MA: Chronic neutrophilic leukemia and chronic myelomonocytic leukemia: WHO defined. Best Pract Res Clin Haematol 19: 571-593, 2006.

8. Sakka V, Tsiodras S, Giamarellos-Bourboulis EJ and Giamarellou H: An update on the etiology and diagnostic evaluation of a leukemoid reaction. Eur J Intern Med 17: 394-398, 2006.

9. Melo JV: The diversity of BCR-ABL fusion proteins and their relationship to leukemia phenotype. Blood 88: 2375-2384, 1996.

10. Pane F, Frigeri F, Sindona M, et al: Neutrophilic-chronic myeloid leukemia: a distinct disease with a specific molecular marker (BCR/ABL with C3/A2 junction). Blood 88: 2410-2414, 1996.

11. Klampfl T, Gisslinger H, Harutyunyan AS, et al: Somatic mutations of calreticulin in myeloproliferative neoplasms. N Engl J Med 369: 2379-2390, 2013.

12. Nangalia J, Massie CE, Baxter EJ, et al: Somatic CALR mutations in myeloproliferative neoplasms with nonmutated JAK2. N Engl J Med 369: 2391-2405, 2013.

13. Ortiz-Cruz K, Amog-Jones G and Salvatore JR: Chronic neutrophilic leukemia with JAK2 gene mutation. Commun Oncol 9: 127-131, 2012.

14. Maxson JE, Gotlib J, Pollyea DA, et al: Oncogenic CSF3R mutations in chronic neutrophilic leukemia and atypical CML. N Engl J Med 368: 1781-1790, 2013.

15. Elliott MA and Tefferi A: Chronic neutrophilic leukemia 2014: Update on diagnosis, molecular genetics and management. Am J Hematol 89: 651-658, 2014.

16. Terré C, Garcia I, Bastie JN, et al: A case of chronic neutrophilic leukemia with deletion (11)(q23). Cancer Genet Cytogenet 110: 70-71, 1999

17. Chen Y, Wang S and Wang W: Deletion of chromosome 17 as a novel cytogenetic finding in chronic neutrophilic leukemia: A case report. Oncol Lett 5: 1704-1706, 2013.

18. Frank MB, Norwood TH and Willerford DM: Chimeric del20q in a case of chronic neutrophilic leukemia. Am J Hemat 64: 229-231, 2000. 
19. Cohen PR: Sweet's syndrome - a comprehensive review of an acute febrile neutrophilic dermatosis. Orphanet J Rare Dis 2: 34, 2007.

20. Ratzinger G, Burgdorf W, Zelger BG and Zelger B: Acute febrile neutrophilic dermatosis: a histopathologic study of 31 cases with review of literature. Am J Dermatopathol 29: 125-133, 2007.

21. Gan GG, Pasagna JF, Eow GI and Nadarajan VS: Chronic neutrophilic leukaemia. Singapore Med J 48: e74-e76, 2007.

22. Castanet J, Lacour JP, Garnier G, et al: Neutrophilia dermatosis associated with chronic neutrophilia leukemia. J Am Acad Dermatol 29: 290-292, 1993

23. Zittoun R, Réa D, Ngoc LH and Ramond S: Chronic neutrophilic leukemia. A study of four cases. Ann Hematol 68: 55-60, 1994.

24. Shigekiyo T, Miyagi J, Chohraku M, et al: Bleeding tendency in chronic neutrophilic leukemia. Int J Hematol 88: 240-242, 2008

25. Kako S, Kanda Y, Sato T, et al: Early relapse of JAK2 V617F-positive chronic neutrophilic leukemia with central nervous system infiltration after unrelated bone marrow transplantation. Am J Hematol 82: 386-390, 2007.

26. Noguchi T, Ikeda K, Yamamoto K, et al: Severe bleeding tendency caused by leukemic infiltration and destruction of vascular walls in chronic neutrophilic leukemia. Int J Hematol 74: 437-441, 2001.

27. Elliott MA, Hanson CA, Dewald GW, Smoley SA, Lasho TL and Tefferi A: WHO-defined chronic neutrophilic leukemia: a long-term analysis of 12 cases and a critical review of the literature. Leukemia 19: 313-317, 2005.
28. Goto H, Hara T, Tsurumi H, Tanabashi S and Moriwaki H: Chronic neutrophilic leukemia with congenital Robertsonian translocation successfully treated with allogeneic bone marrow transplantation in a young man. Intern Med 48: 563-567, 2009.

29. Pardanani A and Tefferi A: Imatinib targets other than bcr/abl and their clinical relevance in myeloid disorders. Blood 104: 1931-1939, 2004.

30. Apperley JF, Gardembas M, Melo JV, et al: Response to imatinib mesylate in patients with chronic myeloproliferative diseases with rearrangements of the platelet-derived growth factor receptor beta. N Eng1 J Med 347: 481-487, 2002.

31. Choi IK, Kim BS, Lee KA, et al: Efficacy of imatinib mesylate (STI571) in chronic neutrophilic leukemia with $\mathrm{t}(15 ; 19)$ : case report. Am J Hematol 77: 366-369, 2004.

32. Elliott MA, Dewald GW, Tefferi A and Hanson CA: Chronic neutrophilic leukemia (CNL): a clinical, pathologic and cytogenetic study. Leukemia 15: 35-40, 2001.

33. Amato D, Memon S and Wang C: Myeloblastic transformation of chronic neutrophilic leukaemia. Br J Haematol 142: 148, 2008. 